Quim. Nova, Vol. 36, No. 5, 628-633, 2013

\title{
CONSTITUINTES FENÓLICOS E ATIVIDADE ANTIOXIDANTE DA GEOPRÓPOLIS DE DUAS ESPÉCIES DE ABELHAS SEM FERRÃO AMAZÔNICAS
}

\author{
Ellen Cristina Costa da Silva*, Magno Perêa Muniz e Rita de Cássia Saraiva Nunomura \\ Departamento de Química, Instituto de Ciências Exatas, Universidade Federal do Amazonas, Av. Gal. Rodrigo O. J. Ramos, 3000 , \\ 69077-040 Manaus - AM, Brasil \\ Sergio Massayoshi Nunomura \\ Departamento de Produtos Naturais, Instituto Nacional de Pesquisas da Amazônia, CP 2223, 69080-971 Manaus - AM, Brasil \\ Gislene Almeida Carvalho Zilse \\ Departamento de Biodiversidade, Instituto Nacional de Pesquisas da Amazônia, CP 2223, 69080-971 Manaus - AM, Brasil
}

Recebido em 13/6/12; aceito em 4/12/12; publicado na web em 12/3/13

\begin{abstract}
PHENOLIC CONSTITUENTS AND ANTIOXIDANT ACTIVITY OF GEOPROPOLIS FROM TWO SPECIES OF AMAZONIAN STINGLESS BEES. We investigated the phenolic constituents and antioxidant activity of geopropolis from two species of stingless Amazonian bees, Melipona interrupta and Melipona seminigra. The chemical investigation of geopropolis from Melipona interrupta led to the isolation of 5,7,4'-trihydroxyflavonone, 3,5,6,7,4'-pentahydroxyflavonol, naringenine-4'- $O$ - $\beta$-D-glucopyranoside and myricetin-3-O- $\beta$-D-glucopyranoside. Their structures were assigned based on spectroscopic analyses, including two-dimensional NMR techniques. Antioxidant activity of methanol and ethanol extracts of $M$. interrupta and M. seminigra were measured using the 1,2-diphenyl-2-picryl-hydrazyl (DPPH) free radical scavenging assay. This is also the first work reporting the chemical investigation of stingless bee species from the Amazonian region.
\end{abstract}

Keywords: flavonoides; geopropolis; Melipona.

\section{INTRODUÇÃO}

A geoprópolis é um produto resinoso produzido pelas abelhas, inclusive pelas espécies Melipona interrupta e Melipona seminigra, a partir da coleta de resinas vegetais, óleos essenciais, pólen, cera e açúcares, que é utilizado na construção e calafetação da colmeia. ${ }^{1,2}$ Tem cor variada de verde, vermelho para marrom escuro, apresenta um aroma característico e propriedades adesivas, porque interage fortemente com óleos e proteínas da pele. ${ }^{3}$

Tem sido utilizada na medicina popular há milhares de anos, devido às propriedades biológicas que a própolis tem, tais como, anti-inflamatória, antibacteriana, ${ }^{4,5}$ antifúngica, ${ }^{6}$ antioxidante,,${ }^{7-9}$ hepato-protetora, antiviral, capacidade de aumentar a resistência natural do corpo a infecções e tratar úlcera gastroduodenal. ${ }^{10}$ Vários estudos registraram que a atividade biológica da própolis depende da composição química, a qual também depende da diversidade geográfica e da variedade genética da rainha. ${ }^{11}$

Como a própolis apresenta várias atividades biológicas, em sua composição química constam muitos produtos naturais bioativos como polifenóis, flavonoides e ésteres do ácido cafeico. ${ }^{12,13}$ É extremamente difícil identificar qual a substância responsável por sua atividade terapêutica, uma vez que existe uma diversidade muito grande de componentes presentes na própolis. Alguns autores afirmam que sua eficácia estaria justamente na composição química heterogênea. ${ }^{14}$ Entre os grupos biologicamente ativos mais importantes descritos na própolis, estão os compostos fenólicos, onde podemos destacar os ácidos gálico, vanílico, ferúlico, $p$ -cumárico, éster fenetílico do ácido cafeico (CAPE), ácidos cafeico e siringíco, que são comuns em produtos apícolas. ${ }^{15}$ Outro grupo de compostos fenólicos encontrado na própolis são os flavonoides. Dentre eles podemos destacar alguns flavonóis: galangina, campeferol, quercetina, miricetina, algumas flavonas: crisina, apigenina,

*e-mail: ellensilva@yahoo.com.br luteolina, tectocrisina, e as flavanonas: pinocembrina, isosacuranetina, naringenina. ${ }^{10}$

A composição química da própolis é influenciada pela flora local e pelas características geográficas e climáticas. Estudos anteriores sobre a própolis brasileira têm demonstrado que existe uma variação muito grande na composição, tanto qualitativa como quantitativa dos compostos fenólicos. ${ }^{16} \mathrm{~A}$ variação qualitativa da composição pode ser observada em análises feitas por cromatografia gasosa acoplada à espectrometria de massas - CG-MS, onde foram identificados diterpenos em amostras de própolis do Mediterrâneo e do Brasil, ${ }^{17,18} \mathrm{e}$ isoflavonas em amostras do Brasil. ${ }^{19}$ Ácidos fenólicos e flavonoides foram identificados por cromatografia líquida acoplada à espectrometria de massas (LC-MS) em amostras de própolis do Brasil e da China ${ }^{6,20}$ Em uma análise por cromatografia líquida de alta eficiência (CLAE) foram identificados flavonoides em amostras de própolis do México. ${ }^{21}$ Benzofenonas preniladas e flavonoides foram isolados em amostras de própolis do Brasil e da India, ${ }^{5,22,23}$ respectivamente.

A atividade biológica e a composição química da própolis têm sido estudadas em vários países e principalmente no Brasil. ${ }^{12,13,16}$ Poucos são os estudos da composição química da própolis de abelhas na Região Amazônica. Existe apenas um estudo da composição química e atividade antibacteriana da própolis da espécie de abelha de Apis mellifera oriunda da cidade de Manaus, ${ }^{5}$ mas nenhuma informação foi encontrada sobre a geoprópolis das espécies de abelhas de Melipona interrupta Latreille, 1811 e Melipona seminigra Friese, 1903 (Apidae, Meliponini) nativas da Região Amazônica. Entretanto, existem informações de trabalhos relatando composição química e atividade biológica da geoprópolis da espécie $M$. interrupta e a avaliação dos constituintes nutricionais do mel e pólen dessa espécie coletada em outras regiões do Brasil..23,24 Outros estudos também têm evidenciado que existe influência da sazonalidade na atividade antibacteriana da própolis da espécie de Apis mellifera proveniente das regiões nordeste e sudeste, provavelmente devido à alteração na concentração de compostos bioativos oriundos das fontes vegetais 
dessas própolis. ${ }^{26}$ Assim, o fator localização geográfica associado à comercialização do extrato em etanol da própolis pela população da região motivou-nos a explorar a atividade antioxidante dos extratos da geoprópolis de duas espécies de Melipona e ao estudo da composição química do extrato da geoprópolis de $M$. interrupta coletada em diferentes localidades do Amazonas.

Considerando a importância de ter compostos fenólicos como um dos principais componentes antioxidantes, o presente trabalho teve como objetivo isolar substâncias fenólicas do extrato em metanol de geoprópolis da espécie de $M$. interrupta, Mi5, cultivada no Instituto Nacional de Pesquisas da Amazônia - INPA pelo Grupo de Pesquisas em Abelhas - GPA, e Mi6, cultivada na comunidade do Ramal do Brasileirinho em Manaus.

\section{PARTE EXPERIMENTAL}

\section{Procedimentos experimentais gerais}

Os espectros uni- e bi-dimensionais foram obtidos no espectrômetro de modelo Unity Inova, marca Varian $\left({ }^{1} \mathrm{H}: 500 \mathrm{MHz} ;{ }^{13} \mathrm{C}\right.$ : $125 \mathrm{MHz}$ ), do Centro de Biotecnologia da Amazônia (CBA). Foram utilizados tetrametilsilano (TSM) como padrão interno e metanol deuterado como solvente. Para as análises por espectrometria de massas com ionização de electrospray (ESI-MS), 1 mg da substância isolada foi diluída em $1 \mathrm{~mL}$ de metanol e injetada diretamente no espectrômetro. Os espectros de massas foram obtidos no modo negativo em um espectrômetro de massas modelo LCQ Fleet, Thermo Scientific (serial: LCF 10151), da Central Analítica da Universidade Federal do Amazonas (UFAM). Foi utilizado metanol deuterado como solvente. Para os fracionamentos por cromatografia líquida em coluna utilizou-se como fase estacionária sílica gel 60 (Merck) (partículas com 0,063-0,2 mm) e sílica gel 60 de fase reversa (Merck) (partículas com 0,040-0,063 mm), tendo como suporte colunas de vidro cilíndricas com dimensões variando de acordo com a quantidade de amostra a ser analisada. A cromatografia em camada delgada comparativa (CCDC) foi empregada para análise e reunião das frações obtidas por cromatografia em coluna. Para o isolamento de substâncias, a cromatografia em camada delgada preparativa (CCDP) foi empregada, para a qual foram utilizadas placas de vidro e a fase estacionária foi preparada com uma suspensão de sílica gel $\mathrm{PF}_{254} 7749$ (Merck) em água. As substâncias em análise foram evidenciadas pelo uso de radiação ultravioleta sob os comprimentos de onda de 254 e 366 $\mathrm{nm}$, pela impregnação das placas em cubas de vidro saturadas por vapores de iodo, pela aspersão com solução de anisaldeído, seguida de aquecimento e pela aspersão com solução de difenilboriloxietilamina (NP) seguida da solução de polietilenoglicol (PEG), revelador para os flavonoides.

\section{Coleta da geoprópolis}

As amostras de geoprópolis das espécies $M$. interrupta e $M$. seminigra foram coletadas diretamente em colmeias alojadas em caixas padronizadas em meliponários do Grupo de Pesquisas em Abelhas (INPA) em 15/07/2011, na comunidade Boa União (Boa Vista do Ramos) em 14/04/2008 e na comunidade do Ramal do Brasileirinho (Manaus) em 22/05/2008. As coletas foram feitas pela equipe do Grupo de Pesquisas em Abelhas do Instituto Nacional de Pesquisas da Amazônia (GPA/INPA).

\section{Extração e isolamento dos flavonoides}

Parte das amostras da geoprópolis de $M$. interrupta e $M$. seminigra foi pulverizada e submetida à extração contínua sólido-líquido em aparelho do tipo soxhlet com metanol, resultando nos extratos em metanol, após evaporação do solvente sob pressão reduzida. Outra parte das amostras da geoprópolis desses dois espécimes foi submetida à maceração a frio com etanol $70 \%$, durante 1 semana, conforme descrição das comunidades que o comercializam. Esses extratos foram preparados para avaliação do seu potencial antioxidante. Dos extratos em metanol testados contra atividade antioxidante, dois apresentaram maior atividade (Mi5 - INPA e Mi6 - Ramal do Brasileirinho). Os extratos brutos Mi5 (10 g) e Mi6 (30 g) foram submetidos à partição líquido-líquido por ordem crescente de polaridade de solventes (hexano, $\mathrm{CHCl}_{3}$ e AcOEt). Após evaporação do solvente de todas as fases em rotaevaporador, obteve-se para Mi5 609,3 mg da fase hexânica, $507,9 \mathrm{mg}$ da fase em $\mathrm{CHCl}_{3}$ e 2,011 g da fase em AcOEt. Para Mi6 obteve-se 5,33 $\mathrm{g}$ da fase hexânica, 2,70 $\mathrm{g}$ da fase em $\mathrm{CHCl}_{3}$ e 9,30 $\mathrm{g}$ da fase em AcOEt.

A fase em clorofórmio (500 mg) do extrato Mi5 foi submetida à cromatografia em coluna de sílica gel 60 utilizando como sistema de eluição dois gradientes: hexano/AcOEt (inicialmente com hexano $40 \%$ ) e $\mathrm{CHCl}_{3} / \mathrm{MeOH}$ (inicialmente com $\mathrm{CHCl}_{3} 100 \%$ ). As amostras obtidas foram analisadas em CCDC e reunidas de acordo com seus fatores de retenção (Rf), resultando em 10 frações. A fração reunida 4 (15,2 mg), coletada em $\mathrm{CHCl}_{3} / \mathrm{MeOH}(9,5: 0,5)$, mostrou-se com três manchas por análise por CCDC e, então, foi submetida à uma separação cromatográfica em camada delgada preparativa (CCDP), usando $\mathrm{CHCl}_{3} / \mathrm{MeOH}(9,5: 0,5)$ como eluente, obtendo-se a naringenina $(1,11,5 \mathrm{mg})$. A fração reunida $6(23,2 \mathrm{mg})$, coletada em $\mathrm{CHCl}_{3}$ / $\mathrm{MeOH}(9,0: 1,0)$, forneceu a 3,5,6,7,4'-penta-hidroxiflavononol (aromadendrina, 2, 3,1 mg) depois de submetida à CCDP, utilizando $\mathrm{CHCl}_{3} / \mathrm{MeOH}(9,0: 1,0)$ como eluente.

A fase em acetato de etila (2,70 g) do extrato Mi5 foi submetida à cromatografia em coluna filtrante em sílica gel 60 (RP-18) utilizando sistema de eluição isocrático com $\mathrm{MeOH} / \mathrm{H}_{2} \mathrm{O}$ (5:5), a fração resultante $\mathbf{1}(1,3 \mathrm{~g})$ sofreu centrifugação $\left(18^{\circ} \mathrm{C}, 10.000 \mathrm{rpm}, 8 \mathrm{~min}\right)$. A fase sobrenadante $(1,2 \mathrm{~g})$ foi submetida a uma cromatografia em coluna de sílica gel 60 (RP-18) utilizando o sistema de fase móvel gradiente $\mathrm{MeOH} / \mathrm{H}_{2} \mathrm{O}$ (inicialmente com $\mathrm{H}_{2} \mathrm{O}$ 60\%). As amostras obtidas através deste procedimento foram analisadas em CCDC e reunidas de acordo com seus Rfs, resultando em 6 frações. A fração reunida 5 (180 $\mathrm{mg}$ ) apresentou um precipitado branco que foi submetido a várias lavagens com $\mathrm{MeOH}$, obtendo-se a naringenina-4'O-ß-glicopiranosídeo (3, 10,2 mg).

A fase em acetato de etila (3,0 g) do extrato Mi6 foi submetida a uma coluna cromatográfica em sílica gel 60 eluindo-se com $\mathrm{CHCl}_{3}$ / $\mathrm{MeOH}$ (gradiente). As amostras obtidas foram então analisadas em CCDC e reunidas de acordo com seus Rfs, resultando em 9 frações. As frações reunidas 7 e $8(740,4 \mathrm{mg})$ apresentaram um precipitado branco. Essas foram submetidas a lavagens com $\mathrm{MeOH}$, gerando 350 mg da substância 3 e um sobrenadante. O sobrenadante (347,8 mg) foi submetido a uma separação cromatográfica em coluna de fase reversa utilizando gel de sílica tipo RP-18 com sistema gradiente de eluição da mistura $\mathrm{MeOH} / \mathrm{H}_{2} \mathrm{O}$ (iniciando com $\mathrm{H}_{2} \mathrm{O} 50 \%$ e terminando com $\mathrm{MeOH} 100 \%$ ). As amostras obtidas foram analisadas em CCDC e reunidas de acordo com seus Rfs, resultando em 11 frações. A fração reunida $6(17,5 \mathrm{mg})$ forneceu a miricetina-3-O- $\beta$-glicopiranosídeo (4, 4,3 mg) após lavagens com $\mathrm{MeOH}$.

\section{Análise dos fenólicos totais}

A quantificação do teor de fenólicos totais nos extratos da geoprópolis, realizada em triplicata, foi determinada pelo método de FolinCiocalteau. ${ }^{27}$ Utilizou-se auxílio da curva analítica de ácido gálico $(31,25-500 \mu \mathrm{g} / \mathrm{mL})$ apresentando regressão linear com confiança de $99,96 \%$. Para obtenção dos resultados foram misturados $200 \mu \mathrm{L}$ 
da amostra $(1,0 \mathrm{mg} / \mathrm{mL})$ com $1,5 \mathrm{~mL}$ do reagente Folin-Ciocalteau (diluído 10 vezes). Após 5 min foram adicionados à mistura 1,5 da solução de bicarbonato de sódio (60 g/L), sendo a reação mantida na ausência de luz por 90 min. A concentração final das amostras após a mistura dos reagentes passou para $62 \mu \mathrm{g} / \mathrm{mL}$. Ao término da reação foi realizada a leitura das absorbâncias utilizando o comprimento de onda de $725 \mathrm{~nm}$ (espectofotômetro UV/Vis Femto 800XI). Os resultados foram expressos em equivalentes de ácido gálico (mg EAG/g de extrato seco). ${ }^{27}$

\section{Capacidade redutora de ferro (FRAP - ferric reducing antioxidant power)}

A capacidade redutora de ferro (FRAP) foi determinada de acordo com o método descrito na literatura. ${ }^{28} \mathrm{O}$ ensaio consiste em preparar uma solução complexante de $\mathrm{Fe}^{3+}$ (reagente FRAP) na proporção 1:1:10 misturando-se $2,5 \mathrm{~mL}$ de solução de TPTZ (2,4,6- tripiridil-1,3,5-triazina) $10 \mathrm{mM}$ e $2,5 \mathrm{~mL}$ de solução de cloreto férrico $20 \mathrm{mM}$ em $25,0 \mathrm{~mL}$ de solução tampão de acetato de sódio $0,25 \mathrm{M}, \mathrm{pH}=3,5$. Foram misturados $100 \mu \mathrm{L}$ da amostra na concentração de $1 \mathrm{mg} / \mathrm{mL}$ em $300 \mu \mathrm{L}$ de água mili-Q, em seguida adicionaram-se 3,0 mL do reagente FRAP. A concentração final das amostras após a mistura dos reagentes passou para $62 \mu \mathrm{g} / \mathrm{mL}$. Após 4 min de incubação, a redução do complexo de $\mathrm{Fe}^{3+}$ para $\mathrm{Fe}^{2+}$ foi obtida através da leitura da absorbância no comprimento de onda a 593 nm (espectrofotômetro UV/Vis Femto 800XI). O procedimento foi realizado em triplicata e os valores de $\mathrm{Fe}^{+2}$ encontrados foram obtidos por meio da equação de regressão linear y $=0,0013 \mathrm{x}$ 0,0947, com coeficiente de correlação de 0,9985 , resultante da construção da curva analítica de sulfato ferroso nas concentrações de 125 a $2000 \mu \mathrm{M}$. Os valores de FRAP foram expressos em $\mu \mathrm{mol}$ de $\mathrm{Fe}(\mathrm{II}) / \mathrm{mg}$ extrato seco. ${ }^{28}$

\section{Ensaio de sequestro de radicais DPPH}

A atividade antioxidante pelo método de sequestro de radicais livres dos extratos foi avaliada segundo o método descrito na literatura. ${ }^{29} \mathrm{O}$ ensaio consistiu em adicionar $1 \mathrm{~mL}$ do radical livre DPPH (2,2-difenil-picril-hidrazila) na concentração de $0,2 \mathrm{mg} / \mathrm{mL}$ nas diluições adequadas de cada extrato (variando entre 6,25-890 $\mu \mathrm{g}$ / $\mathrm{mL}$ ), obtendo as absorbâncias após $30 \mathrm{~min}$ na ausência de luz em um espectrofotômetro no comprimento de onde de $518 \mathrm{~nm}$. As absorbâncias obtidas foram convertidas em porcentagem da atividade antioxidante (AA\%) segundo a equação:

$$
\mathrm{AA} \%=100-\left\{\left[\left(\mathrm{Abs}_{\text {amostra }}-\mathrm{Abs}_{\text {branco }}\right) \times 100\right] / \mathrm{Abs}_{\text {controle }}\right\}
$$

Os resultados foram calculados a partir da construção de uma curva analítica para cada amostra da porcentagem da atividade antioxidante versus concentração do extrato, onde através da equação de regressão linear resultante foi calculada a concentração capaz de sequestrar 50\% $\left(\mathrm{CS}_{50}\right)$ dos radicais livres de DPPH. Foi utilizado como padrão positivo o flavonoide quercetina. ${ }^{29}$

\section{5,7,4'-Tri-hidroxi-flavanona (1, Naringenina)}

Cristais amarelados. ESI-MS, $m / z$ : $271[\mathrm{M}-\mathrm{H}]-$. RMN ${ }^{1} \mathrm{H}[500$ $\mathrm{MHz}, \mathrm{CD}_{3} \mathrm{OD}, \delta$ (ppm)]: 5,33 (dd, $J=13,0$ e 3,0 Hz, H-2), 3,10 (dd, $J=17,0$ e 13,0 Hz, H-3a), 2,69 (dd, $J=17,0$ e 3,0 Hz, H-3b), 5,89 (d, $J=2,0 \mathrm{~Hz}, \mathrm{H}-6$ ), 5,88 (d, $J=2,0 \mathrm{~Hz}, \mathrm{H}-8$ ), 7,30 (d, $J=8,5 \mathrm{~Hz}, \mathrm{H}-2$ ' e H-6'), 6,81 (d, J= 8,0 Hz, H-3'-H-5'). RMN ${ }^{13} \mathrm{C}\left[125 \mathrm{MHz}, \mathrm{CD}_{3} \mathrm{OD}\right.$, $\delta$ (ppm)]: 197,7 (C-4), 168,3 (C-9), 165,4 (C-7), 164,8 (C-5), 159,01 (C-4'), 131,0 (C-1'), 129,02 (C-2' e C-6'), 116,33 (C-3' e C-5'), 103,3 (C-10), 80,47 (C-2), 97,05 (C-8), 96,16 (C-6), 44,02 (C-3). 3,5,6,7,4'-penta-hidroxi-flavonol (2, Aromadendrina)

Sólido amarelado. $\mathrm{RMN}{ }^{1} \mathrm{H}$ [500 MHz, $\mathrm{CD}_{3} \mathrm{OD}, \delta$ (ppm)]: 4,96 (d, $J=11,5 \mathrm{~Hz}, \mathrm{H}-2), 4,52$ (d, $J=11,5 \mathrm{~Hz}, \mathrm{H}-3$ ), 5,85 (s, H-8), 7,35 (d, $J=8,5$ Hz, H-2' e H-6'), 6,83 (d, $J=8,5$ Hz, H-3'-H-5'). RMN ${ }^{13} \mathrm{C}$ [125 MHz, CD $\left.30 D, \delta(p p m)\right]: ~ 159,19$ (C-4'), 129,41 (C-1'), 130,33 (C-2' e C-6'), 116,13 (C-3' e C-5'), 106,17 (C-10), 84,91 (C-2), 96,72 (C-8), 73,61 (C-3).

\section{Naringenina-4'-O- $\beta$-Glicopiranosídeo (3)}

Sólido branco amorfo. ESI-MS, $m / z: 433$ [M-H] $]^{-}, 271 . \mathrm{RMN}^{1} \mathrm{H}$ [500 MHz, CD $\mathrm{OD}_{3} \delta$ (ppm)]: 5,41 (dd, $J=12,5$ e 3,0 Hz, H-2), 3, 10 (dd, $J=17,0$ e $12,5 \mathrm{~Hz}, \mathrm{H}-3 \mathrm{a}), 2,74$ (dd, $J=17,0$ e $3,0 \mathrm{~Hz}, \mathrm{H}-3 \mathrm{~b}$ ), $5,91$ (d, $J=2,0 \mathrm{~Hz}, \mathrm{H}-6), 5,89$ (d, $J=2,0 \mathrm{~Hz}, \mathrm{H}-8$ ), 7,43 (d, $J=8,5$ Hz, H-2' e H-6'), 7, 14 (d, $J=7,0$ Hz, H-3'-H-5'), 4,90 (d, $J=8,5$ Hz, H-1"), 3,47 (d, J=3,0 Hz, H-2"), 3,46 (d, $J=3,0 \mathrm{~Hz}, \mathrm{H}-3$ "), 3,48 (m, H-4"), 3,45 (m, H-5"), 3,70 (dd, J = 12,0 e 6,0 Hz, H-6"), 3,91 (dd, $J=12,0$ e $2,0 \mathrm{~Hz}, \mathrm{H}-6$ "). RMN ${ }^{13} \mathrm{C}\left[125 \mathrm{MHz}, \mathrm{CD}_{3} \mathrm{OD}, \delta(\mathrm{ppm})\right]$ : 197,4 (C-4), 168,3 (C-9), 165,4 (C-7), 164,7 (C-5), 159,2 (C-4'), 134,1 (C-1'), 128,79 (C-2' e C-6'), 117,84 (C-3' e C-5'), 103,3 (C-10), 80,13 (C-2), 97,12 (C-8), 96,21 (C-6), 44,03 (C-3), 102,21 (C-1”), 74,91 (C-2"), 77,99 (C-3"), 71,38 (C-4”), 78,19 (C-5”), 62,52 (C-6”).

\section{Miricetina-3-O- $\beta$-Glicopiranosídeo (4)}

Cristais verdes. ESI-MS, m/z: 479 [M-H] ${ }^{-}, 317 . \mathrm{RMN}{ }^{1} \mathrm{H}[500$ $\mathrm{MHz}, \mathrm{CD}_{3} \mathrm{OD}, \delta$ (ppm)]: 6,39 (d, $\left.J=2,0 \mathrm{~Hz}, \mathrm{H}-6\right), 6,19$ (d, $J=2,0$ Hz, H-8), 7,33 (s, H-2' e H-6'), 4,70 (d, J = 8,0 Hz, H-1'), 3,46 (m, H-2"), 3,43 (m, H-3"), 3,53 (m, H-4"), 3,36 (m, H-5"), 3,77 (dd, $J=$ 12,0 e 4,0 Hz, H-6"), 3,86 (dd, $J=12,0$ e 2,0 Hz, H-6").

\section{RESULTADOS E DISCUSSÃO}

A investigação das frações em $\mathrm{CHCl}_{3}$ e em AcOEt, provenientes do fracionamento cromatográfico do extrato Mi5, resultou no isolamento e caracterização das flavanonas naringenina (1), naringenina glicosilada (3) e do flavonol aromadendrina (2). O fracionamento cromatográfico da fração em AcOEt proveniente do extrato Mi6 da geoprópolis também resultou no isolamento da flavanona naringenina glicosilada (3) e do flavonol miricetina glicosilada (4).

Os espectros de RMN de ${ }^{1} \mathrm{H}$ dos compostos 1 [M-H] 433,3 [M$\mathrm{H}]^{-} 271$ e 4 [M-H]- 479 apresentaram sinais para um grupo hidroxila $(\mathrm{OH})$ quelado e um par de dupletos na região de hidrogênios aromáticos com constantes de acoplamento para hidrogênios meta correlacionados $(J=2 \mathrm{~Hz})$, típico de flavonoides com anel "A" substituídos nas posições 5 e 7, sugerindo que estes flavonoides são 5,7-di-hidroxi, os dados estão de acordo com a literatura e estruturas analisadas. ${ }^{30}$

Os espectros de RMN de ${ }^{1} \mathrm{H}$ das flavanonas 1 e $\mathbf{3}$ mostram o sistema $\mathrm{ABX}$ na região de hidrogênios alifáticos (1: $\delta 5,33 \mathrm{dd}, \mathrm{H}-2$; 3,10 dd, H-3a; 2,69 dd H-3b e 3: $\delta$ 5,41 dd, H-2; 3,10 dd, H-3a; 2,74 dd, H-3b) para o anel "C". Para as flavanonas $\mathbf{1}$ e $\mathbf{3}$ e para o flavonol 2 há a existência no anel "B" do sistema AA'BB' com os hidrogênios H-2'e H-6'equivalentes em 1: $\delta 7,30(\mathrm{~d}, J=8,5 \mathrm{~Hz}), 2$ : $\delta 7,35$ (d, $J=$ 8,5) e 3: $\delta 7,43(\mathrm{~d}, J=8,5 \mathrm{~Hz})$ e os H-3'e H-5' equivalentes em 1: $\delta$ $6,81(\mathrm{~d}, J=8,0 \mathrm{~Hz}), 2: \delta 6,83(\mathrm{~d}, J=8,5)$ e $3: \delta 7,14(\mathrm{~d}, J=7,0 \mathrm{~Hz})$, acoplando em orto, típicos de anel "B" $p$-dissubstituído. Os espectros de $\mathrm{RMN}$ de ${ }^{13} \mathrm{C}$ exibem dois grupos oxi metínicos (1: $\delta$ 80,47 C-2 e 44,02 C-3 e 3: $\delta$ 80,13 C-2 e 44,03 C-3), caracterizando o esqueleto flavanona. Assim, foram identificados naringenina (1) e naringenina4'-O- $\beta$-glicopiranosídeo (3), cujos dados espectroscópicos estão de acordo com os valores da literatura. ${ }^{30}$

Amostras de própolis são conhecidas por apresentarem o flavonoide naringenina, que apresenta atividades anti-inflamatória e antioxidante. ${ }^{8,31} \mathrm{~A}$ atividade anti-inflamatória é resultante da supressão da síntese de prostaglandinas e de leucotrienos pelos 
macrófagos. ${ }^{31}$ A presença de naringenina também foi confirmada em análises por CLAE com os extratos aquoso, em etanol, em acetona e em clorofórmio de própolis oriunda de outros países como China e Itália. ${ }^{7,8}$ Ao mesmo tempo, são conhecidas informações da identificação de naringenina em análises por CG-MS em amostras de própolis do Peru. ${ }^{32}$ Para a naringenina glicosilada não existem informações na literatura.

Na região de açúcares do espectro de $\mathrm{RMN}$ de ${ }^{1} \mathrm{H}$ das substâncias 3 e 4 pôde-se identificar a presença de dois monossacarídeos. Cada substância apresenta hidrogênios anoméricos com deslocamentos em $\delta 4,90$ e $J=8,5$ e $\delta 4,70$ com $J=8,0$, respectivamente. A presença de um dupleto em cada substância indicou a configuração $\beta$ para os carbonos anoméricos C-1". A presença dos hidrogênios H-6", par de dupletos, com sinais de deslocamentos químicos em 3: $\delta 3,91$ (dd, 12 e $2,0 \mathrm{~Hz})$ e $\delta 3,70(\mathrm{dd}, 12$ e $6,0 \mathrm{~Hz})$ e $4: \delta 3,86(\mathrm{dd}, 12$ e $2,0 \mathrm{~Hz})$ e $\delta 3,77$ (dd, 12 e 4,0 Hz) na região de hidrogênios metilênicos no espectro de RMN de ${ }^{1} \mathrm{H}$ evidenciou a presença da parte do açúcar.

O esqueleto do flavonol 4 foi sugerido pelo singleto em $\delta 7,33(\mathrm{H}-$ 2" e H-6") no espectro de RMN de ${ }^{1} \mathrm{H}$, indicando as posições 3', 4'e 5' como tri-hidroxi no anel "B". Também a ausência de hidrogênios alifáticos nas posições 2 e 3 do anel " $\mathrm{C}$ ", no espectro de RMN de ${ }^{1} \mathrm{H}$, indicou a presença de um sistema de carbonos $\alpha, \beta$-insaturados do flavonol 4, cujos dados de $\mathrm{RMN}$ de ${ }^{1} \mathrm{H}$ estão de acordo com os valores indicados na literatura. ${ }^{30,33} \mathrm{~A}$ posição da unidade glicosídica na posição C-3 do flavonol 4 foi deduzida pela diferença nos deslocamentos químicos dos carbonos C-2 e C-3 do glicosídeo em comparação com os da aglicona miricetina, ${ }^{33}$ sendo assim possível indicar a miricetina3-O- $\beta$-glicopiranosídeo (4). Existem informações da identificação da aglicona miricetina em análises por CLAE em amostras de própolis da China. ${ }^{7}$ Mas não existem relatos da identificação de miricetina glicosilada em amostras de própolis..$^{33}$

$\mathrm{O}$ espectro de RMN de ${ }^{1} \mathrm{H}$ do flavonol 2 mostra um singleto com sinal em $\delta 5,85$ na região de hidrogênios aromáticos, típico de flavonoides com anel "A" substituídos nas posições 5, 6 e 7, ou seja, flavonoides 5,6,7-tri-hidroxi. Também foi possível verificar nos espectros de RMN de ${ }^{1} \mathrm{H}$ o sistema $\mathrm{AB}$ na região de hidrogênios alifáticos em 2: $\delta 4,96(\mathrm{~d}, J=11,5 \mathrm{~Hz}, \mathrm{H}-2)$ e $\delta 4,52(\mathrm{~d}, J=11,5 \mathrm{~Hz}$, H-3) para o anel "C". Os deslocamentos químicos de hidrogênio para a substância aromadendrina (2) estão de acordo com os valores de RMN de ${ }^{1} \mathrm{H}$ indicados na literatura. ${ }^{30,34} \mathrm{Na}$ literatura há informações sobre o isolamento do flavonol 7-metil-éter-aromadendrina em amostras do ninho da espécie de abelha Trigona spinipes. ${ }^{35}$

Pela estrutura das substâncias isoladas, observou-se que os extratos de própolis de $M$. interrupta apresentam substâncias glicosiladas, algo que não é muito comum em amostras de própolis de outras regiões, ${ }^{10,15}$ percebe-se assim a influência da origem do material vegetal coletado por essa espécie de abelha. A seletividade dessa espécie também é evidenciada uma vez que se isolou a mesma substância, naringenina-4'-O- $\beta$-glicopiranosídeo (3), dos extratos de geoprópolis coletados em diferentes localizações da cidade de Manaus (Figura 1).

\section{Fenóis totais}

Os antioxidantes, que em muitos casos apresentam estruturas fenólicas, são utilizados no organismo, principalmente, para retardar ou prevenir processos oxidativos, impedindo a formação de radicais livres. ${ }^{36}$ É necessário mensurar os compostos fenólicos presentes nas amostras de geoprópolis em estudo para a realização posterior dos testes antioxidantes. A análise quantitativa dos compostos fenólicos nos extratos em metanol e em etanol de própolis das espécies Melipona interrupta (Mi) e Melipona seminigra (Ms) é mostrada nas Tabelas 1 e 2 e na Figura 2.

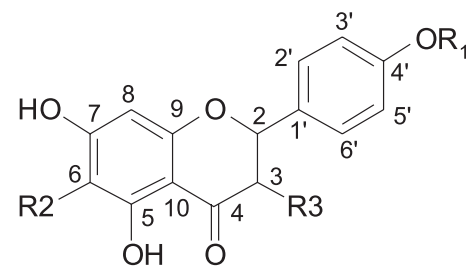

$$
\begin{aligned}
& \text { 1: } R_{1}=H, R_{2}=H, R_{3}=H \\
& \text { 2: } R_{1}=H, R_{2}=O H, R_{3}=O H \\
& \text { 3: } R_{1}=\text { glicose, } R_{2}=H, R_{3}=H
\end{aligned}
$$<smiles>[R]Oc1c(-c2cc(O)c(O)c(O)c2)oc2cc(O)cc(O)c2c1=O</smiles>

4: $\mathrm{R}=$ glicose

Figura 1. Substâncias isoladas de M. interrupta

Tabela 1. Fenólicos totais e atividade antioxidante dos extratos em metanol da própolis (EMP) de $M$. interrupta (Mi) e M. seminigra (Ms)

\begin{tabular}{cccc}
\hline EMP & $\begin{array}{c}\text { Fenólicos totais } \\
(\mu \mathrm{g} \text { "EAG/g EMP })\end{array}$ & $\begin{array}{c}\text { FRAP } \\
(\mathrm{mmol} \mathrm{Fe}(\mathrm{II}) / \mathrm{mg} \mathrm{EMP})\end{array}$ & $\begin{array}{c}\mathrm{DPPH} \\
\mathrm{CS}_{50}(\mu \mathrm{g} / \mathrm{mL})\end{array}$ \\
\hline Mi2 & $431,0 \pm 2,9$ & $6,0 \pm 2,4$ & $493,3 \pm 1,9$ \\
Mi3 & $828,3 \pm 3,1$ & $15,8 \pm 2,4$ & $131,4 \pm 1,9$ \\
Mi4 & $3563,5 \pm 1,8$ & $63,2 \pm 0,0$ & $13,1 \pm 3,0$ \\
Mi5 & $5199,4 \pm 0,5$ & $106,6 \pm 0,5$ & $10,0 \pm 0,5$ \\
Mi6 & $2193,1 \pm 1,8$ & $34,6 \pm 0,9$ & $20,5 \pm 1,5$ \\
Ms1 & $616,0 \pm 3,4$ & $7,0 \pm 4,2$ & $115,7 \pm 0,4$ \\
Ms2 & $411,4 \pm 4,7$ & $4,7 \pm 5,0$ & $155,6 \pm 3,2$ \\
Ms3 & $1607,6 \pm 3,6$ & $6,0 \pm 3,9$ & $234,3 \pm 4,0$ \\
Ms4 & $1197,2 \pm 1,2$ & $11,0 \pm 1,8$ & $129,2 \pm 3,0$ \\
Ms5 & $622,5 \pm 2,1$ & $5,2 \pm 5,0$ & $161,0 \pm 3,3$ \\
\hline
\end{tabular}

Os valores são expressos em média $\pm \mathrm{CV}$ (coeficiente de variação) obtido a partir de análises em triplicata $(\mathrm{n}=3)$. * EAG - Equivalente em ácido gálico.

Tabela 2. Fenólicos totais e atividade antioxidante dos extratos em etanol da própolis (EEP) de $M$. interrupta (Mi) e M. seminigra (Ms)

\begin{tabular}{cccc}
\hline EEP & $\begin{array}{c}\text { Fenólicos totais } \\
\left(\mu \mathrm{g}{ }^{*} \text { EAG/g EMP }\right)\end{array}$ & $\begin{array}{c}\text { FRAP } \\
(\mathrm{mmol} \mathrm{Fe}(\mathrm{II}) / \mathrm{mg} \mathrm{EMP})\end{array}$ & $\begin{array}{c}\text { DPPH } \\
\mathrm{CS}_{50}(\mu \mathrm{g} / \mathrm{mL})\end{array}$ \\
\hline Mi2 & $419,3 \pm 0,91$ & $3,3 \pm 2,3$ & $>500,0 \pm 2,9$ \\
Mi3 & $628,0 \pm 1,7$ & $5,4 \pm 1,2$ & $216,0 \pm 0,9$ \\
Mi4 & $3334,3 \pm 0,9$ & $40,0 \pm 0,4$ & $17,1 \pm 4,9$ \\
Mi5 & $4378,0 \pm 0,2$ & $52,0 \pm 0,0$ & $10,0 \pm 0,5$ \\
Mi6 & $2861,0 \pm 0,6$ & $31,4 \pm 0,7$ & $26,3 \pm 3,9$ \\
Ms1 & $634,3 \pm 5,0$ & $5,4 \pm 1,2$ & $169,0 \pm 0,8$ \\
Ms2 & $748,0 \pm 2,6$ & $6,5 \pm 3,6$ & $141,0 \pm 4,4$ \\
Ms3 & $1413,0 \pm 1,5$ & $4,8 \pm 4,7$ & $288,0 \pm 5,0$ \\
Ms4 & $973,0 \pm 0,2$ & $11,0 \pm 5,0$ & $325,0 \pm 0,1$ \\
Ms5 & $503,0 \pm 0,6$ & $3,8 \pm 3,8$ & $>500,0 \pm 5,0$ \\
\hline
\end{tabular}

Os valores são expressos em média $\pm \mathrm{CV}$ (coeficiente de variação) obtido a partir de análises em triplicata $(\mathrm{n}=3)$ * EAG - Equivalente em ácido gálico. 

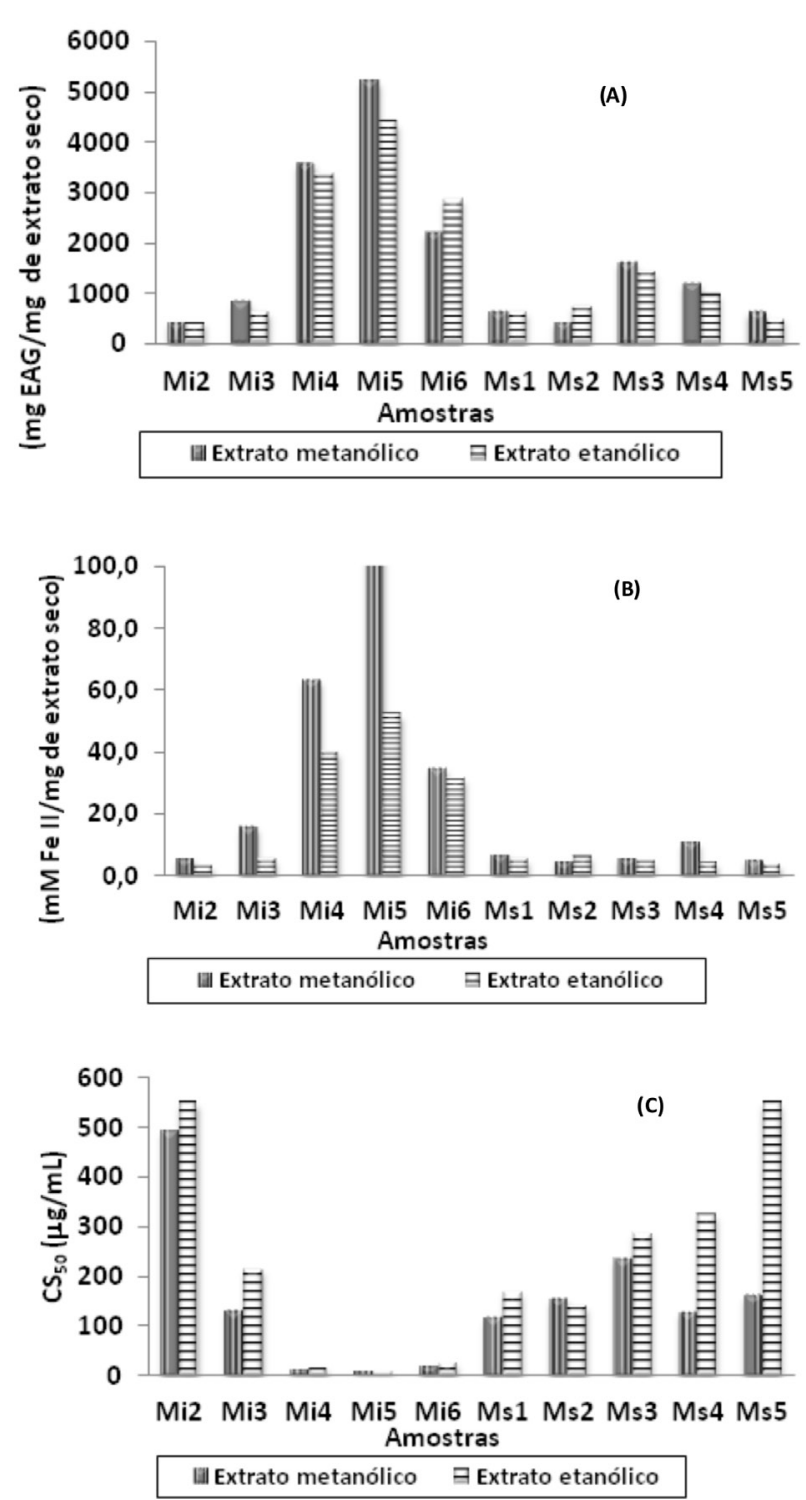

Figura 2. Análises dos extratos metanólicos e etanólicos de própolis de Melipona interrupta (Mi) e Melipona seminigra (Ms). (A) Fenólicos totais; atividade antioxidante: (B) FRAP, (C) capacidade de sequestrar $50 \%$ dos radicais livres de $\mathrm{DPPH}\left(\mathrm{CS}_{50}\right)$

Não houve discrepância nos valores de fenólicos obtidos tanto em metanol $100 \%$ como em etanol $70 \%$. Foi observada maior concentração de fenólicos nos extratos em metanol Mi4, Mi5 e Mi6 com 3564, 5199, $2193 \mathrm{mg}$ EAG/g de extrato seco, e nos extratos em etanol Mi4, Mi5 e Mi6 com 3334, 4378, 2861 mg EAG/g de extrato seco, respectivamente. Os extratos da espécie $M$. seminigra apresentaram baixos teores de compostos fenólicos, sendo um indicativo de pouca atividade antioxidante. Como não houve discrepância significativa nos valores encontrados nos extratos em metanol e em etanol de $M$. interrupta, pode-se afirmar que tanto metanol como etanol podem extrair substâncias fenólicas. Os valores encontrados estão, para ambas as espécies, em uma faixa superior aos valores encontrados (200-400 mg/g) para extratos de própolis da China (aquoso), ${ }^{7}$ Itália com 293-356 mg/g (em $\mathrm{CHCl}_{3}$, em EtOH e em acetona) e Índia com 159-269 mg/g (aquosos e em EtOH). ${ }^{8,23}$ Os extratos das espécies de abelhas amazônicas confirmam a influência da variabilidade da composição química e origem do material no resultado.

\section{Capacidade redutora de ferro (FRAP - ferric reducing} antioxidant power)

Neste ensaio, quanto maior a capacidade do extrato de reduzir o $\mathrm{Fe}^{3+}$ para $\mathrm{Fe}^{2+}$, mais intensa é a mudança da cor verde-amarelada para tons de azul, devido à presença de redutores (antioxidantes) que promovem a conversão do complexo- $\mathrm{Fe}^{3+}$ à forma ferrosa $\left(\mathrm{Fe}^{2+}\right)$. Ao se medir a formação da intensidade da cor azul no comprimento de onda de $593 \mathrm{~nm}$, é possível medir a concentração de complexo- $\mathrm{Fe}^{2+}$ formado. Nesse sentido, quanto maior for a absorbância das amostras a $593 \mathrm{~nm}$, maior o poder de redução. As propriedades de redução são geralmente associadas com a presença de redutores, que têm mostrado exercer ação antioxidante pela quebra da reação em cadeia dos radicais livres por doação de um átomo de hidrogênio. ${ }^{37} \mathrm{O}$ poder de redução dos extratos foi analisado na concentração de $1,0 \mathrm{mg} /$ $\mathrm{mL}$. As melhores capacidades redutoras foram obtidas nos mesmos extratos que apresentaram maiores valores de compostos fenólicos, com resultados que variaram de 34,6 a 106,6 mmol Fe II/mg de extrato seco nos extratos em metanol e 31,4 a 52,0 6 mmol Fe II/mg de extrato seco nos extratos em etanol (Tabelas 1 e 2, Figura 2). As amostras Mi4, Mi5 e Mi6 dos dois extratos mostraram maior capacidade de reduzir $\mathrm{Fe}^{3+}$ a $\mathrm{Fe}^{2+}$, podendo reagir com os radicais livres estabilizando e bloqueando as reações em cadeias radicalares. Esses testes associados ao ensaio de DPPH corroboram como indicativo de extratos com atividade antioxidante. Os valores encontrados para ambos os extratos das duas espécies apresentaram altas concentrações de fenólicos, com valores acima da faixa $(0,20-3,47 \mu \mathrm{mol})$ dos valores encontrados para extratos aquosos de própolis da China. ${ }^{7}$ A maioria dos extratos de ambas as espécies também apresentou valores acima da faixa $(0,41-0,77 \mathrm{mmol})$ dos valores dos extratos em $\mathrm{CHCl}_{3}$, em EtOH e em acetona de própolis da Itália. ${ }^{8}$ Em comparação aos valores encontrados para extratos em etanol e em etileno glicol de própolis da Espanha, as duas espécies apresentaram valores superiores. ${ }^{38}$

\section{Ensaio de sequestro de radicais DPPH.}

O método de sequestro de radicais livres é um dos mecanismos mais conhecidos e um dos mais utilizados como teste padrão para avaliar a atividade antioxidante de compostos específicos ou extratos. O radical DPPH tem a característica específica de absorver em 518 nm (cor púrpura), e que em contato com substâncias sequestradoras (antioxidantes) de radicais livres ocasiona o descoramento, através do fornecimento de átomos de hidrogênio ou por doação de elétrons, diminuindo significativamente a absorbância. Portanto, quanto menor a absorbância em $518 \mathrm{~nm}$, maior será a atividade do extrato em sequestrar os radicais de DPPH..$^{39}$ Os valores da atividade antioxidante são mostrados nas Tabelas 1 e 2 e Figura 2. Esses valores são expressos em valores de $\mathrm{CS}_{50}$, que avalia a concentração dos extratos de própolis necessária para sequestrar 50\% dos radicais livres de DPPH. Os resultados são comparados ao controle positivo (quercetina) que apresentou $\mathrm{CS}_{50}=4,0 \mu \mathrm{g} / \mathrm{mL}$. Os extratos metanólicos Mi4, Mi5 e Mi6 novamente apresentaram atividade antioxidante mais expressiva, com capacidade de sequestrar 50\% dos radicais livres de DPPH na concentração de 13,10 e $20 \mu \mathrm{g} / \mathrm{mL}$, respectivamente, e com 17, 10 e $26 \mu \mathrm{g} / \mathrm{mL}$ nos extratos em etanol. Assim observou-se uma possível correlação entre a presença de compostos fenólicos e a atividade antioxidante dos extratos (Figura 2).

Os valores da atividade antioxidante dos extratos Mi4, Mi5 e Mi6 de $M$. interrupta foram superiores aos valores encontrados para extratos aquosos de própolis da China, ${ }^{7}$ da Itália $\left(\mathrm{em}^{\mathrm{CHCl}}{ }_{3}\right.$, em EtOH e em acetona) e da Índia, frente a amostras de extratos aquosos e em EtOH. ${ }^{8,23}$ Os valores encontrados no resultado dos extratos das duas espécies de abelhas confirmam o potencial de atividade antioxidante 
desses extratos de própolis. A atividade antioxidante é influenciada pela alta presença de compostos fenólicos, os quais foram indicados pelo ensaio de fenólicos totais. Outro fator determinante é a influência da variabilidade da composição química e origem do material coletado por cada espécie.

\section{CONCLUSÃO}

A presença de compostos fenólicos é considerada responsável pela atividade antioxidante e estatisticamente os valores da $\mathrm{CS}_{50}$ estão relacionados com substâncias fenólicas totais presentes nas amostras..$^{40-42}$ Tal correlação pôde ser observada nos resultados obtidos dos ensaios de fenólicos totais e dos testes antioxidantes dos extratos em metanol e em etanol da própolis. Pela análise de CCD comparativa percebeu-se que os mesmos componentes extraídos em metanol também são encontrados quando extraídos em etanol, garantindo que o extrato de própolis comercial é eficiente. Entre os extratos de geoprópolis das duas espécies, as amostras de M. interrupta apresentaram maior teor de compostos fenólicos e maior atividade antioxidante em relação aos extratos de geoprópolis produzida por M. seminigra. Portanto, as espécies vegetais visitadas por abelhas $M$. interrupta apresentam classes de substâncias que proporcionam a atividade antioxidante.

As análises dos extratos da geoprópolis dessas espécies mostraram resultados promissores, pois apresentaram atividades (antioxidantes) importantes para a manutenção e prevenção de doenças, além de ser utilizado como alimento (nutracêutico) pela população local. Do fracionamento dos extratos metanólicos da geoprópolis de $M$. interrupta com maior atividade antioxidante foi possível isolar quatro flavonoides.

\section{AGRADECIMENTOS}

Às instituições de fomento à pesquisa CNPq e FAPEAM pelos auxílios financeiros, ao CBA pelos espectros de RMN, ao INPA pela infraestrutura e ao Programa de Pós-Graduação em Química - PPGQ-UFAM.

\section{REFERÊNCIAS}

1. Carvalho-Zilse, G. A.; Nunes-Silva, C. G.; Threats to the Stingless Bees in the brazilian Amazon: How to deal with scarce biological data and an increasing rate of destruction; Em Bees: Biology, Threats and Colonies; Florio, R. M., ed.; Nova Science Publishers: New York, 2012.

2. Nogueira-Neto, P.; Vida e criação de abelhas indígenas sem ferrão, Wiley: São Paulo, 1997.

3. Sforcin, J. M.; J. Ethnopharmacol. 2007, 113, 1.

4. Murad, J. M.; Calvi, S. A.; Soares, A. M. V. C.; Bankova, V.; Sforcin, J. M.; J. Ethnopharmacol. 2002, 79, 331.

5. Ishida, V. F. C.; Negri, G.; Salatino, A.; Bandeira, M. F. C. L.; Food Chem. 2011, 125, 966

6. Yang, S. Z.; Peng, L. T.; Su, X. J.; Chen, F.; Cheng, Y. J.; Fan, G.; Pan, S. Y.; Food Chem. 2011, 127, 210.

7. Guo, X.; Chen, B.; Luo, L.; Zhang, X.; Dai, X.; Gong, S.; J. Agric. Food Chem. 2011, 59, 12610.

8. Papotti, G.; Bertelli, D.; Bortolotti, L.; Plessi, M.; J. Agric. Food Chem. 2012, 60, 2852.

9. Miguel, M. G.; Nunes, S.; Dandlen, S. A.; Cavaco, A. M.; Antunes, M. D.; Adv. Environ. Biol. 2011, 5, 345.

10. Castaldo, S.; Capasso, F.; Fitoterapia 2002, 73, S1.

11. Lustosa, S. R.; Galindo, A. B.; Nunes, L. C. C.; Randau, K. P.; Rolim-Neto, P. J.; Rev. Bras. Farmacogn. 2008, $18,447$.
12. Banskota, A. H.; Teruka, Y.; Adnyana, I. K.; Midosikawa, M.; Matsushige, K; Kadota, S.; Phytomedicine 2001, 8, 16.

13. Nagaoka, T.; Banskota, A. H.; Midorikawa, K.; Matsushige, K.; Kadota, S.; Biol. Pharm. Bull. 2003, 26, 487.

14. Matsuno, T.; Z. Naturforsch. 1995, 50, 93.

15. Soares, S. E.; Rev. Nut. 2002, 15, 71.

16. Park, Y. K.; J. Agric. Food Chem. 2004, 52, 1100.

17. Popova, M.; Trusheva, B.; Antonova, D.; Cutajar, S.; Mifsud, D.; Farrugia, C.; Tsvetkova, I.; Najdenski, H.; Bankova, V.; Food Chem. 2011, 126,1431

18. Nunes, C. A.; Guerreiro, M. C.; J. Sci. Food Agric. 2012, 92, 433.

19. Alencar, S. M.; Oldoni, T. L. C.; Castro, M. L.; Cabral, I. S. R.; CostaNeto, C. M.; Cury, J. A.; Rosalen, P. L.; Ikegaki, M.; J. Ethnopharmacol. 2007, 113, 278.

20. Carvalho, A. A.; Finger, D.; Machado, C. S.; Schmidt, E. M.; Costa, P. M.; Alves, A. P. N. N.; Morais, T. M. F.; Queiroz, M. G. R.; Quináia, S. P.; Rosa, M. R.; Santos, J. M. T.; Pessoa, C. P.; Moraes, O.; CostaLotufo, L. V.; Sawaya, A. C. H. F.; Eberlin, M. N.; Torres, Y. R.; Food Chem. 2011, 126, 1239.

21. Valencia, D.; Alday, E.; Robles-Zepeda, R.; Garibay-Escobar, A.; Galvez-Ruiz, J. C.; Salas-Reyes, M.; Jiménez-Estrada, M.; VelazquezContreras, E.; Hernandez, E.; Velazquez, C.; Food Chem. 2012, 131, 645.

22. Castro, M. L.; Nascimento, A. M.; Ikegaki, M.; Costa-Neto, C. M.; Alencar, S. M.; Rosalen, P. L.; Biorg. Med. Chem. 2009, 17, 5332.

23. Laskar, R. A.; Sk, I.; Roy, N.; Begun, N. A.; Food Chem. 2010, 122, 233.

24. Bankova, V.; Christov, R.; Popov, S.; Marcucci, M. C.; Tsvetkova, I.; Kujumgiev, A.; Fitoterapia 1999, 70, 190.

25. Souza, R. C.; Yuyama, L. K. O.; Aguiar, J. P. L.; Oliveira, F. P. M.; Acta Amaz. 2004, 34, 333.

26. Castro, M. L.; Cury, J. A.; Rosalen, P. L.; Alencar, S. M.; Ikegaki, M.; Duarte, S.; Koo, H.; Quim. Nova 2007, 30, 1512.

27. Velioglu, Y. S.; Mazza, G.; Gao, L.; Oomah, B. D.; J. Agric. Food Chem. 1998, 46, 4113.

28. Luximon-Ramman, B. T.; Soobratee, M. A.; Aruoma, O. I.; J. Agric. Food Chem. 2002, 50, 5042.

29. Mensor, L. L.; Menezes, F. S.; Leitão, G. G.; Reis, A. S.; Santos, T. C.; Coube, C. S.; Leitão, S. G.; Phytother. Res. 2001, 15, 127.

30. Markham, K. R.; Geiger, H. Em The Flavonoids: Advances in research since 1986; Harborne, J. B., ed.; Chapman \& Hall: London, 1994, p. 441.

31. Mirzoeva, O. K.; Calder, P. C.; Prostaglandins Leukot. Essent. Fatty Acids 1996, 55, 441.

32. Seven, I.; Aksu, T.; Seven, P. T.; Lives.Prod. Sci. 2012, 148, 10.

33. Ceruks, M.; Romoff, P.; Fávero, O. A.; Lago, J. H. G.; Quim. Nova 2007, $30,597$.

34. Almeida, S. C. X.; Lemos, T. L. G.; Silveira, E. R.; Loiola, O. D.; Quim. Nova 2005, 28, 57.

35. Freitas, M. O.; Ponte, F. A. F.; Lima, M. A. S.; Silveira, E. R.; J. Braz. Chem. Soc. 2008, 19, 532.

36. Degáspari, C. H.; Waszczynskyj, N.; Propriedades Antioxidantes de Compostos Fenólicos, 5 a ed., Wiley: Curitiba, 2004.

37. Shimada, K.; Fujikawa, K.; Yahara, K.; Nakamura, T.; J. Agric. Food Chem. 1992, 40, 945.

38. Bonvehí, J. S.; Gutiérrez, A. L.; J. Am. Oil Chem. Soc. 2011, 88, 1387.

39. Amarowicz, R.; Pegg, R. B.; Rahimi-Moghaddam, P.; Barl, B.; Weil, J. A.; Food Chem. 2004, 84, 551.

40. Kumazawa, S.; Hamasaka, T.; Nakayama, T.; Food Chem. 2004, 84, 329.

41. Pereira, J. A.; Oliveira, I.; Sousa, A.; Ferreira, I.; Bento, A.; Estevinho, L.; Food Chem. Toxicol. 2008, 46, 2103.

42. Sousa, A.; Ferreira, I. C. F. R.; Barros, L.; Bento, A.; Pereira, J. A.; LWTFood Sci. Technol. 2008, 41, 739. 